\title{
Study on Chaotic Peculiarities of Magnetic-Mechanical Coupled System of Giant Magnetostrictive Actuator
}

\author{
Hongbo Yan, Enzuo Liu (iD, Pengbo Zhao, Pei Liu, and Rui Cao \\ College of Mechanical Engineering, Inner Mongolia University of Science \& Technology, Baotou 014010, China \\ Correspondence should be addressed to Enzuo Liu; 1030975114@qq.com
}

Received 13 August 2020; Revised 23 September 2020; Accepted 13 November 2020; Published 25 November 2020

Academic Editor: Viktor Avrutin

Copyright $(2020$ Hongbo Yan et al. This is an open access article distributed under the Creative Commons Attribution License, which permits unrestricted use, distribution, and reproduction in any medium, provided the original work is properly cited.

\begin{abstract}
We studied the chaotic peculiarities of magnetic-mechanical coupled system of GMA. Based on the working principle of GMA and according to Newton's second law of motion, first piezomagnetic equation, disk spring design theory, and structural dynamics principle of GMA, the present study established a GMA magnetic-mechanical coupled system model. By carrying out data modeling of this coupled system model, the bifurcation chart of the system with the variation of damping factor, excitation force, and exciting frequency parameters as well as the homologous offset oscillogram, phase plane trace chart, and Poincaré diagram was obtained, and the chaotic peculiarities of the system were analyzed. The influence of parametric errors on the coupled system was studied. The analytical results showed that the oscillation equation of the GMA magnetic-mechanical coupled system had nonlinearity and the movement morphology was complicated and diversified. By adjusting the damping factor, exciting frequency, and excitation force parameters of the system, the system could work under the stable interval, which provided theoretical support for the stability design of GMA.
\end{abstract}

\section{Introduction}

Due to its advantages of large displacement, fast response speed, large magnetic-mechanical coupling coefficient, and high reliability $[1,2]$, the giant magnetostrictive material (GMM) has a broad application prospect in aerospace, national defense and military industry, machinery, precision machining, medical equipment, and other fields [3-8]. The giant magnetostrictive actuator (GMA) is becoming an outstanding representative of new driving mechanism because its core component GMM has superior performance, which can better realize the mutual transformation of magnetic-mechanical energy.

In recent years, many scholars have studied the magnetic-mechanical coupled system. Yoo et al. [9] used the three-dimensional finite element method to carry out analysis of the GMA magnetic-mechanical coupled system and verified the reliability of this method through experiments. Niu et al. [10] proposed a GMA dynamic model based on the J-A model, which considered the magnetic-mechanical coupling effect and could improve the forecast accuracy of GMA output displacement. Ebrahimi et al. [11] established a magnetic-mechanical coupled system model by conducting change calculation of continuous energy function for the minimized system. This model studied the relationship between stress and magnetic conductivity. Yang et al. [12] established a magnetic-mechanical strong coupled system model for the force sensor made by GMM and compared it with the measuring result to examine the effectiveness of the model. Zhao [13] carried out experimental research on the dynamic and static peculiarities of GMM under the impact of a magnetic-mechanical coupled system. Li [14] proposed a GMA hysteresis nonlinear model based on the magnetic-mechanical coupling principle and carried out experimental analysis on the dynamic and static peculiarities of GMA force and output displacement.

However, as a result of the hysteresis nonlinearity in the input and output relation of GMA, it not only makes GMA easy to produce positioning errors in the open-loop system but also causes unstable closed-loop system in complex tracking problems, which seriously affected the adhibition of GMA [15]. Therefore, in order to improve the performance 
and enhance the actual control and application of GMA, enhancing the stability of the GMA nonlinear system has become a hot topic in recent years.

If the GMA magnetic-mechanical coupled system is under the working condition of chaotic movement, the actual control and stability of the GMA nonlinear system will be seriously damaged. Therefore, the study of chaotic peculiarities of the magnetic-mechanical coupled system has occupied a very important position in the stability of the GMA nonlinear system.

Zhu et al. [16] found that under the action of in-plane harmonic wave and random excitation, the giant magnetostrictive thin film shape memory alloy composite cantilever slab system had random bifurcation and chaos phenomena with the change of parameters. Xu et al. [17] discussed that the giant magnetostrictive piezoelectric composite sensor system had multifrequency response in the harmonic magnetic scene, and the system movement changed from periodical orbit to chaos when increasing the external excitation. Bowong and Kakmeni [18] proposed a robust control scheme with simple structure to suppress the chaotic movement of the system. Sarmah and Ananthakrishna [19] established a magneticstrain coupling nonlinear oscillator model, which effectively predicted the chaotic quasiperiodic path of the system. Sun [20] established a mechanical model of a GMA system based on the piezomagnetic theory and analyzed the chaotic phenomenon that may exist in the model. Yan et al. [21] established the mathematical model of a GMA hysteresis oscillatory system and studied the bifurcation and chaos phenomena of the GMA system. At present, there are fewer research achievements of parameters on the chaotic peculiarities of the GMA magnetic-mechanical coupled system and the systematic theoretical basis is insufficient. However, the extensive research has been made on the chaotic peculiarities of other nonlinear systems [22-26].

First of all, based on the GMA working principle, this paper set up the GMA magnetic-mechanical coupled system model and obtained the coupled equation of this system. Then, it used the Melnikov method to analyze the threshold value of chaos phenomenon generated by the system. In addition, on the basis of bifurcation chart, offset oscillogram, phase plane trace chart, and Poincaré diagram, it explained the chaotic peculiarities of this coupled system. Finally, the influence of parametric errors on the coupled system was studied. The research result is beneficial to optimize the stability design and improvement plan of the GMA magnetic-mechanical coupled system.

\section{Working Principle of the GMA}

The GMA working principle is as follows: when the alternating current is implemented in the driving coil, the alternating magnetic scene will be generated. Under the effect of the driving magnetic scene, the GMM rod has extension changes along the direction of the rod, thus generating the output displacement. The bias current is applied in the bias coil, which can generate the bias magnetic field so as to avoid the occurrence of GMA frequency-doubled effect and improve the sensitivity and linear peculiarities of GMA. The pretensioner is composed of an outer wall sleeve, disk spring, upper end cover, and output rod, which can offer some prestressing forces so that the GMM rod can work in an optimal state. The closed magnetic circuit is mainly composed of upper and lower magnetic conductive loops, flux sleeve, and flux sheet, which can lower magnetic leakage and effectively alleviate the jam of magnetic scene to external equipment. Figure 1 shows the GMA structure chart.

\section{GMA Magnetic-Mechanical Coupled System Model}

We can see from the GMA working principle that under the impact of steady-state magnetic scene, the displacement of upper end face of GMM rod is the same as that of the output rod. In the movement process, GMM output force $T$, disk spring prepressure $P(x)$, damping force of output $\operatorname{rod} Q(\dot{x})$, and external excitation $F \cos \omega t$ of output rod have a combined action on the GMA output rod. Its equivalent mechanical model is shown in Figure 2, where $x$ is the displacement of the output rod.

In light of Newton's second law of motion, the dynamic differential equation of the GMA magnetic-mechanical coupled system is as follows:

$$
T-P-Q-F \cos \omega t=m \ddot{x},
$$

where $m$ is the quality of the output rod.

According to the first piezomagnetic equation, when the excitation magnetic scene is uniform to the axial direction of the rod, the simplified piezomagnetic equation along the direction of the rod can be indicated as follows [27, 28] by ignoring the influence of shear stress, transverse stress, and transverse excitation:

$$
\begin{gathered}
\zeta=S_{33}^{H} \sigma+d_{33} H_{3}, \\
B=d_{33} \sigma+\mu_{33}^{T} H_{3},
\end{gathered}
$$

where $\zeta, \sigma$, and $H_{3}$ are the strain intensity, stress intensity, and magnetic field intensity of GMM; $d_{33}$ is the piezomagnetic coefficient; $S_{33}^{H}$ is the compliant mechanism; $B$ is the magnetic scene intensity; and $\mu_{33}^{T}$ is the magnetic conductivity.

The GMA system has been analyzed and the output force $T$ of GMM rod is as follows:

$$
T=E A \zeta
$$

where $E$ and $A$ are the resilience modulus and cross-sectional area of GMM rod.

Under the condition of steady-state magnetic scene and ignoring magnetic leakage flux, the magnetic scene strength $\mathrm{H}_{3}$ of GMM is as follows according to the magnetomotive force of magnetic circuit:

$$
H_{3}=\frac{N I}{\mu_{33}^{T} P A}-\frac{d_{33} \sigma}{\mu_{33}^{T}},
$$

where $N$ and $I$ are the count of turns and current of coil and $P$ is the total magnetic resistance of magnetic circuit. 


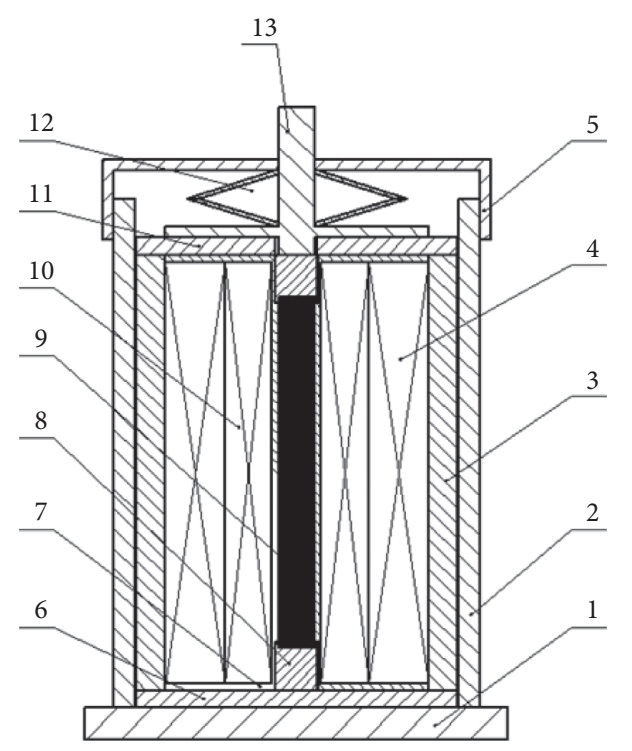

FIGURE 1: GMA structure. 1, base; 2, outer wall socket; 3, flux sleeve; 4 , drive coil; 5 , upper end cover; 6 , lower magnetic conductive ring; 7, coil former; 8 , flux sheet; 9,GMM rod; 10 , bias coil; 11 , upper magnetic conductive ring; 12 , disc ring; 13 , output rod.

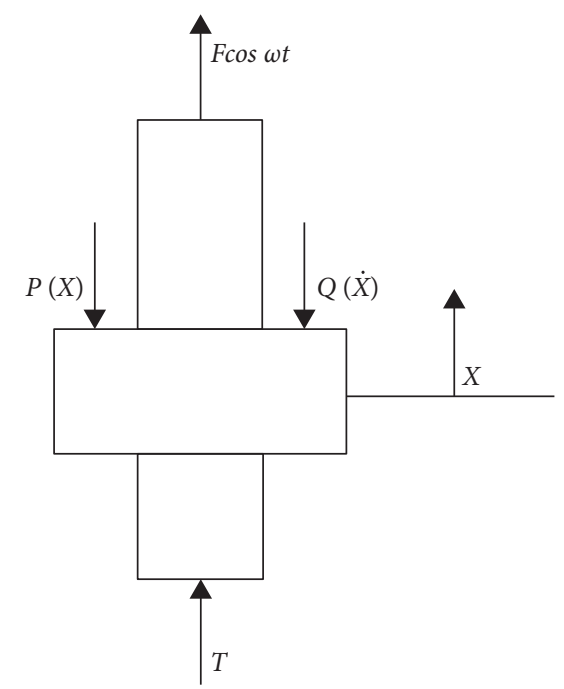

Figure 2: Equivalent mechanical model of GMA output rod.

It is obtained from the simultaneous formulas (2)-(5) that the output force $T$ of GMM rod is

$$
T=E A\left[\left(S_{33}^{H}-\frac{d_{33}^{2}}{\mu_{33}^{T}}\right) \sigma+\frac{d_{33} N I}{\mu_{33}^{T} P A}\right] .
$$

Under the impact of steady-state magnetic scene, the number of turns and current of coil and the cross-sectional area of the GMM rod are all constants, and when the magnetic circuit is the closed circuit, the GMM magnetic resistance is only related to its relative magnetic conductivity and cross-sectional area, so it can be regarded as the constant. Without considering the influence of system magnetic resistance and magnetic scene changes on various parameters [29], the GMM rod's output force $T$ is

$$
T=\frac{E A}{l}\left(1-\frac{E d_{33}^{2}}{\mu_{33}^{T}}\right) x,
$$

where $l$ is the GMM rod's length.

We can see from the disk spring design theory [30] that the prepressure of the disk spring $P$ is

$$
P=\frac{4 E}{1-\mu^{2}} \frac{d^{4}}{K_{1} D^{2}} K_{4}^{2} \frac{f_{z}}{d}\left[K_{4}^{2}\left(\frac{h}{d}-\frac{f_{z}}{d}\right)\left(\frac{h}{d}-\frac{f_{z}}{2 d}\right)+1\right],
$$

where $\mu$ is Poisson's ratio and $d$ and $D$ are the thickness and outer diameter of disk spring. $K_{1}$ and $K_{4}$ are determined by disk spring design handbook. $f_{z}$ is the deformation amount of combination disk spring. If $f_{z}=2 f, f$ is the deformation amount of a single piece disk spring. $f_{z}$ in this paper is taken as $x$, and $h$ is the calculated value of normal deformation amount of disk spring.

It can be obtained through simplifying formula (8):

$$
P=k x+u_{1} x^{2}+u_{2} x^{3}
$$

where

$$
\begin{aligned}
k & =\frac{4 E K_{4}^{4} d^{3}}{K_{1} D^{2}\left(1-\mu^{2}\right)}\left[\frac{1}{K_{4}^{2}}+\left(\frac{h}{d}\right)^{2}\right], \\
u_{1} & =-\frac{6 E K_{4}^{4} h d}{K_{1} D^{2}\left(1-\mu^{2}\right)}, \\
u_{2} & =\frac{2 E K_{4}^{4} d}{K_{1} D^{2}\left(1-\mu^{2}\right)} .
\end{aligned}
$$

Through the analysis of the GMA system, it can be seen that the damping force $Q$ of output rod is as follows:

$$
Q=c \dot{x},
$$

where $c$ is the damping factor of the GMA system.

Formulas (7), (9), and (11) are substituted into formula (1) to obtain the equation of the GMA magnetic-mechanical coupled system:

$$
m \ddot{x}+c \dot{x}+k_{e} x+u_{1} x^{2}+u_{2} x^{3}-F \cos \omega t=0,
$$

where

$$
k_{e}=k+\frac{E A}{l}\left(\frac{E d_{33}^{2}}{\mu_{33}^{T}}-1\right) .
$$

Dividing both sides of formula (12) by $m$, the formula can be sorted out as follows:

$$
\ddot{x}+C \dot{x}+\alpha x+\omega_{0} x^{2}+\beta x^{3}-F_{0} \cos \omega t=0,
$$

where 


$$
\begin{aligned}
C & =\frac{c}{m}, \\
\alpha & =\frac{k_{e}}{m}, \\
\omega_{0} & =\frac{u_{1}}{m}, \\
\beta & =\frac{u_{2}}{m}, \\
F_{0} & =\frac{F}{m} .
\end{aligned}
$$

\section{Chaotic Peculiarity Analysis of GMA Magnetic-Mechanical Coupled System}

When carrying out chaotic response analysis of the GMA magnetic-mechanical coupled system, formula (14) needs to be simplified into the standard form. If $\dot{x}=y, C=\varepsilon r$, $F_{0}=\varepsilon f$, and $0<\varepsilon \ll 1$, the standard form of formula (14) shall be

$$
\left.\begin{array}{l}
\dot{x}=y, \\
\dot{y}=-\alpha x-\omega_{0} x^{2}-\beta x^{3}+\varepsilon(-r \dot{x}+f \cos \omega t) .
\end{array}\right\}
$$

The function is defined as follows:

$$
\begin{aligned}
l(x) & =\alpha x+\omega_{0} x^{2}+\beta x^{3}, \\
m(\dot{x}, \ddot{x}, \omega t) & =-r \dot{x}+f \cos \omega t .
\end{aligned}
$$

When $\varepsilon=0$, formula $(16)$ is

$$
\left.\begin{array}{l}
\dot{x}=y, \\
\dot{y}=-\alpha x-\omega_{0} x^{2}-\beta x^{3} .
\end{array}\right\}
$$

When $\varepsilon=0$, system (18) is the Hamilton system without disturbance, and its Hamilton action shall be

$$
h=\frac{1}{2} y^{2}+\frac{\alpha}{2} x^{2}+\frac{\omega_{0}}{3} x^{3}+\frac{\beta}{4} x^{4}
$$

It can be obtained from formula (18) that its balance points are $\mathrm{O}(0,0), A\left(\left(\left(-\omega_{0}+\sqrt{\omega_{0}^{2}-4 \alpha \omega_{0}}\right) / 2 \beta\right), 0\right)$, and $B$ $\left(\left(\left(-\omega_{0}-\sqrt{\omega_{0}^{2}-4 \alpha \omega_{0}}\right) / 2 \beta\right), 0\right)$.

The featured equation of the system shall be

$$
\lambda^{2}=-\alpha-2 \omega_{0} x^{2}-3 \beta x^{2}
$$

The balance point $(0,0)$ is substituted into formula (20) to obtain $\lambda= \pm i \sqrt{\alpha}$, which are the conjugate complex roots. Therefore, the balance point $\mathrm{O}(0,0)$ is the center. Similarly, the balance point $B\left(\left(\left(-\omega_{0}-\sqrt{\omega_{0}^{2}-4 \alpha \omega_{0}}\right) / 2 \beta\right), 0\right)$ is also the center while $A\left(\left(\left(-\omega_{0}+\sqrt{\omega_{0}^{2}-4 \alpha \omega_{0}}\right) / 2 \beta\right), 0\right)$ is the saddle point. As a result, the system has the homoclinic orbit $I$ that passes through the saddle point $A$ $\left(\left(\left(-\omega_{0}+\sqrt{\omega_{0}^{2}-4 \alpha \omega_{0}}\right) / 2 \beta\right), 0\right)$, as shown in Figure 3 .

Therefore, the parameter equation of homoclinic orbit is

$$
\left.\begin{array}{l}
x(t)= \pm \frac{2 \sqrt{2} \omega_{0} e^{ \pm\left(\omega_{0} t / 3 \sqrt{\beta}\right)}}{3 \beta\left(e^{ \pm\left(2 \omega_{0} t / 3 \sqrt{\beta}\right)}+1\right)}+\frac{\omega_{0}}{3 \beta}, \\
y(t)= \pm \frac{2 \sqrt{2} \omega_{0}^{2}\left(1-e^{ \pm\left(2 \omega_{0} t / 3 \sqrt{\beta}\right)}\right)}{9 \beta^{(5 / 2)}\left(e^{ \pm\left(2 \omega_{0} t / 3 \sqrt{\beta}\right)}+1\right)^{2}} .
\end{array}\right\}
$$

The Melnikov function of homoclinic orbit is

$$
\begin{aligned}
M\left(t_{0}\right) & =\int_{-\infty}^{+\infty} m\left[x(t), \dot{x}(t), \omega\left(t+t_{0}\right)\right] y(t) \mathrm{d} t \\
& =\int_{-\infty}^{+\infty}\left[-r \dot{x}(t)+f \cos \omega\left(t+t_{0}\right)\right] y(t) \mathrm{d} t .
\end{aligned}
$$

Formula (21) is substituted into formula (22) to obtain the integral:

$$
M\left(t_{0}\right)=\frac{4 \omega_{0}^{3} r}{81 \beta^{(5 / 2)}}+\frac{2 \sqrt{2} \pi \omega f e^{-2 \pi \omega \beta^{(1 / 2)} / \omega_{0}} \sin \omega t_{0}}{\beta^{(1 / 2)}\left(1-\cosh \left(3 \pi \omega \beta^{(1 / 2)} / \omega_{0}\right)\right) .}
$$

When $M\left(t_{0}\right)=0$,

$$
\sin \omega t_{0}=-\frac{\sqrt{2} \omega_{0}^{3} r\left(1-\cosh \left(3 \pi \omega \beta^{(1 / 2)} / \omega_{0}\right)\right)}{81 \pi \omega f \beta^{2} e^{-\left(2 \pi \omega \beta^{(1 / 2)} / \omega_{0}\right)}} .
$$

As $\left|\sin \omega t_{0}\right| \leq 1$,

$$
\left|-\frac{\sqrt{2} \omega_{0}^{3} r\left(1-\cosh \left(3 \pi \omega \beta^{(1 / 2)} / \omega_{0}\right)\right)}{81 \pi \omega f \beta^{2} e^{-\left(2 \pi \omega \beta^{(1 / 2)} / \omega_{0}\right)}}\right| \leq 1 .
$$

$r$ and $f$ are substituted into formula (25). According to the Melnikov method, as we can see from $M\left(t_{0}\right)=0$, the threshold condition that may make the system generate chaos in the sense of Smale horseshoe transformation is as follows: 


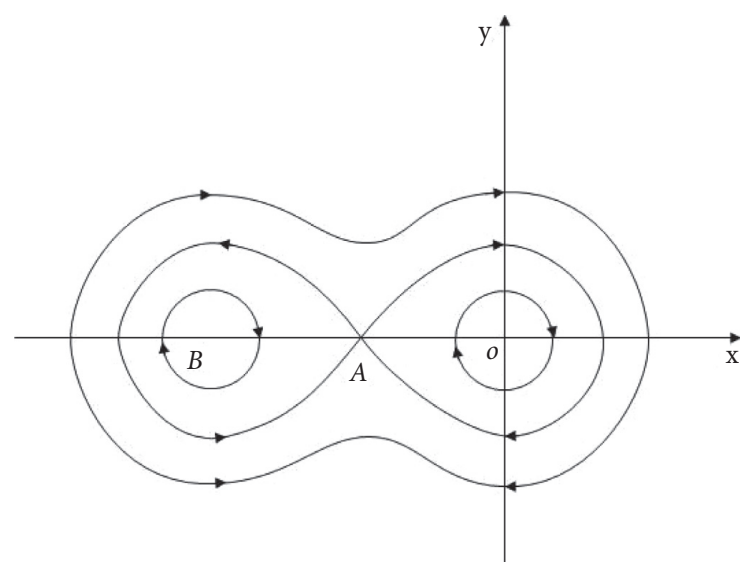

Figure 3: Homoclinic orbit.

$$
\left|\frac{\sqrt{2} \omega_{0}^{3} C\left(1-\cosh \left(3 \pi \omega \beta^{(1 / 2)} / \omega_{0}\right)\right)}{81 \pi \omega F_{0} \beta^{2} e^{-\left(2 \pi \omega \beta^{(1 / 2)} / \omega_{0}\right)}}\right| \leq 1 .
$$

When $\varepsilon$ is small enough, if $M\left(t_{0}\right)$ has a straightforward null point within the range of $t_{0} \in(0, N)$, the system may have the bifurcation of homoclinic orbit, which means that the chaotic behavior may occur.

\section{Numerical Modeling}

For equation (14), each parameter value is shown in Table 1. The fourth-order Runge-Kutta method was used to solve the equation of the GMA magnetic-mechanical coupled system. Based on the numerical solution, the bifurcation chart of the system under different parameters was obtained, and the chaotic peculiarities of the system under the changes of damping factor, excitation force, and exciting frequency were studied combined with the offset oscillogram, phase plane trace chart, and Poincaré diagram.

5.1. The Influence of Damping Factor on the Chaotic Peculiarities of the System. Figure 4 shows the bifurcation chart of the GMA magnetic-mechanical coupled system changing with the damping factor of the system. The initial condition is $[0,0,0.06]$. When the damping factor of the system changes, the path to chaos of the system is as follows:

$$
\begin{aligned}
\text { Chaotic movement } & \longrightarrow \text { intermittent chaos } \\
& \longrightarrow \text { period } 2 \longrightarrow \text { periodic movement }
\end{aligned}
$$

We can see from Figure 4 that when the damping factor of the system is $\in(0.06,0.14)$, the system carried out chaotic movement, as shown in Figure $5(c=0.1)$. When the damping factor of the system is $(0.14,0.32)$, the system degrades into period 2 movement with inverse bifurcation, as shown in Figure $6(c=0.2)$. When the damping factor of the system is 0.2 , the offset oscillogram is regularly distributed and has a constant period. The phase trajectory chart is a closed curve. Poincaré diagram shows two attractive points, so the system carries out a typical doubly
TABLE 1: Each parameter value of the GMA system.

\begin{tabular}{lc}
\hline Parameter & Value \\
\hline Equivalent damping factor of GMA system $C$ & 0.1 \\
Equivalent stiffness coefficient of GMA system $\alpha$ & -0.2 \\
Modulus of secondary rigidity term of disk spring $\omega_{0}$ & 0.1 \\
Modulus of third rigidity term of disk spring $\beta$ & 1 \\
Excitation force of GMA system $F$ & 0.22 \\
Exciting frequency of GMA system $\omega$ & 1.2 \\
\hline
\end{tabular}

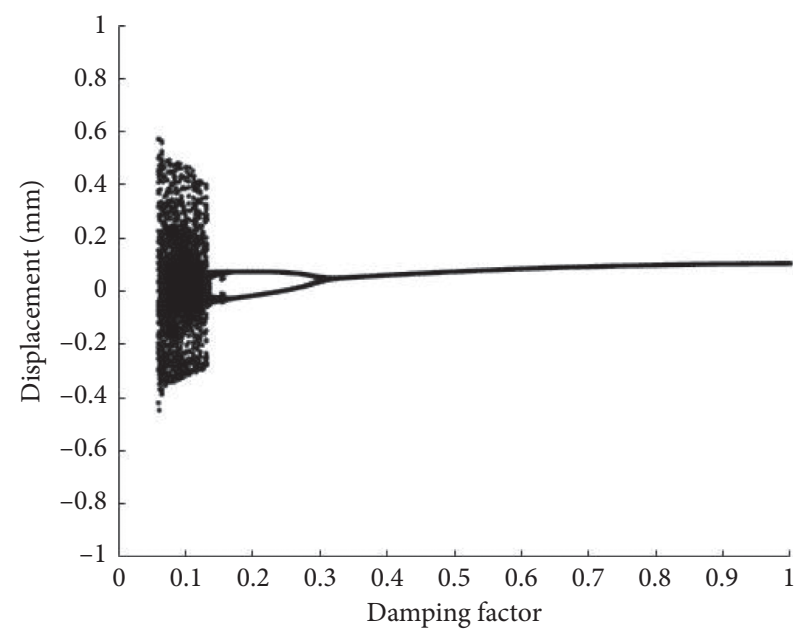

FIgURE 4: The bifurcation chart of the GMA magnetic-mechanical coupled system changing with the damping factor of the system.

periodic movement and has intermittent chaos when the damping factor of the system is around 0.16 . When the damping factor of the system is $\epsilon(0.32,1.00)$, the system carries out the steady-state movement with the period of 1 , as shown in Figure $7(c=0.5)$, that is, when the damping factor of the system is 0.5 , the offset oscillogram is regularly distributed and has a constant period. The phase trajectory chart is a closed curve. Poincaré diagram shows one attractive point, so the system carries out steady-state movement with the single period. Table 2 shows the form of movement of damping factor in different areas.

Figure 5 shows the offset oscillogram, phase trajectory chart, and Poincaré diagram of the system when the damping factor of the system is 0.1 . It can be seen that the offset oscillogram in Figure 5(a) has an irregular distribution with an unstable period. The phase trajectory chart in Figure 5(b) cannot be closed and has filled the phase space area for a long time. The phase trajectory movement is the reciprocating movement with an infinite period. In Figure 5(c), the Poincaré diagram is neither a limited point set nor a closed curve. Therefore, the system can be determined to carry out chaotic movement.

5.2. The Influence of Excitation Force on the Chaotic Peculiarities of the System. Figure 8 shows the bifurcation chart of the GMA magnetic-mechanical coupled system changing with the excitation force. The initial condition is $\left[\begin{array}{lll}0 & 0 & 0.02\end{array}\right]$. When the excitation force alters, the path to chaos of the system is as follows: 


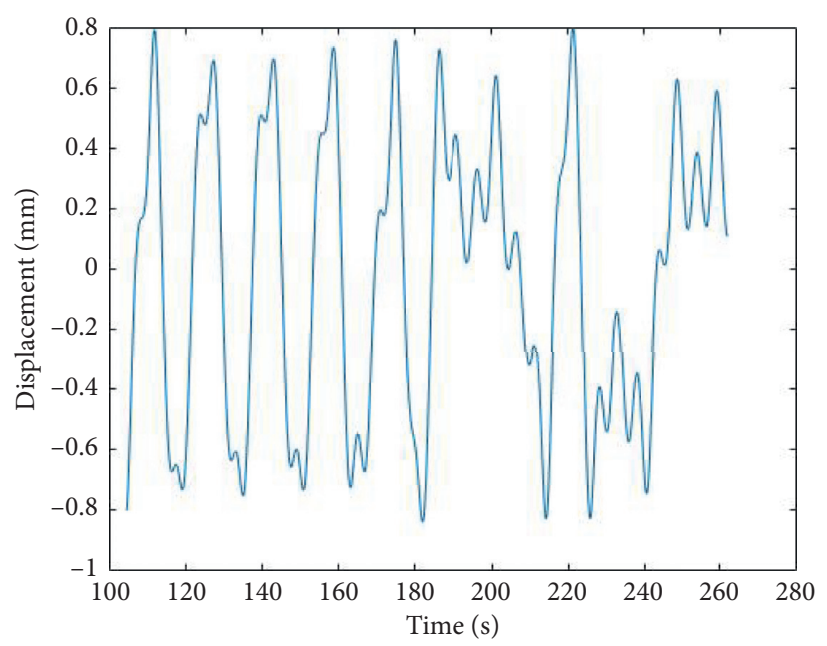

(a)

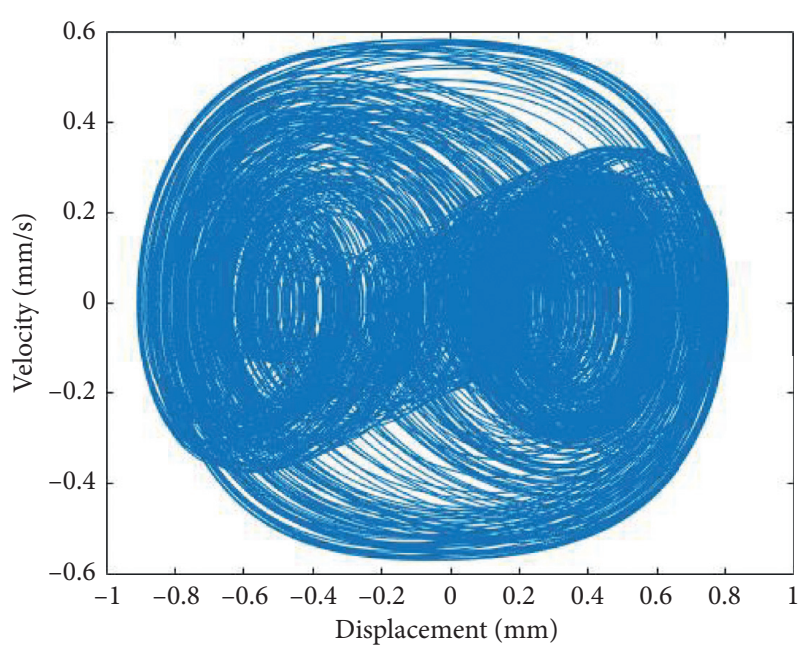

(b)

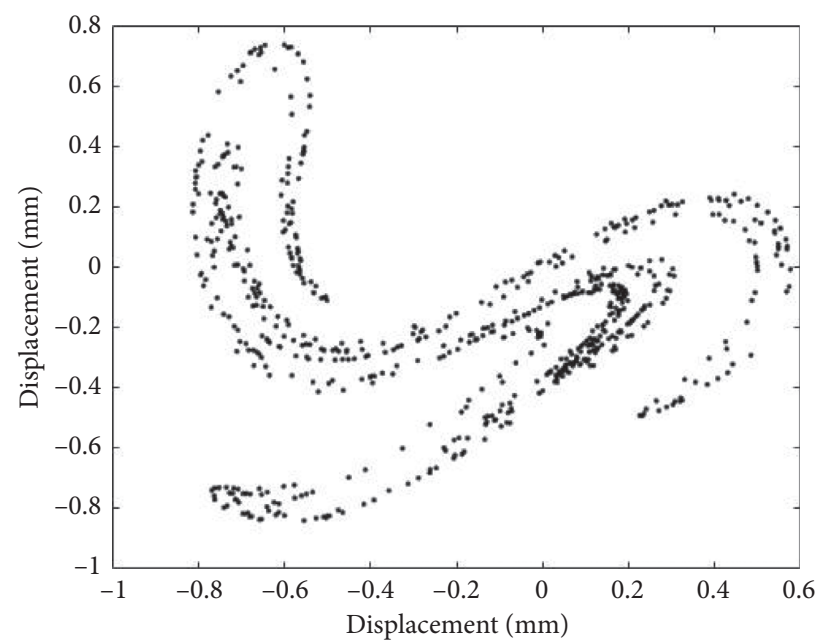

(c)

Figure 5: (a) The offset oscillogram, (b) phase trajectory chart, and (c) Poincaré diagram of the system when $C=0.1$.

Periodic movement $\longrightarrow$ Period $2 \longrightarrow$ chaotic movement $\longrightarrow$ multiple intermittent chaost $\longrightarrow$ period $2 \longrightarrow$ periodic movement

$\longrightarrow$ chaotic movement $\longrightarrow$ multiple intermittent chaos $\longrightarrow$ quasi - periodic movement $\longrightarrow$ periodic movement

We can see from Figure 8 that when the excitation force is $\epsilon(0.02,0.045)$, the system carries out the steady-state movement with the period of 1 . When the excitation force is $\in(0.045$, $0.14)$, the system carries out the movement with the period of 2 accompanied with the tangent bifurcation, as shown in Figure 9 $(F=0.1)$. When the excitation force is 0.1 , the offset oscillogram is regular with a constant period, while the phase trajectory chart is a closed curve. Poincaré diagram shows two attractive points, so the system carries out a typical doubly periodic movement. When the excitation force is $\epsilon(0.14,0.315)$, the system carries out the chaotic movement as shown in Figure 10 $(F=0.3)$ and has multiple intermittent chaos around 0.155 and 0.315 . When the excitation force is $\in(0.315,0.36)$, the system degrades into period 2 movement with inverse bifurcation. When the excitation force is $\epsilon(0.36,0.39)$, the system carries out the movement with the period of 1 . When the excitation force is $\epsilon(0.39,0.59)$, the system carries out the chaotic movement as shown in Figure $11(F=0.4)$ and has multiple intermittent chaos around 0.52 and 0.545 . When the excitation force is $\epsilon(0.59,0.74)$, the system carries out quasiperiodic movement and has multiple intermittent chaos around 0.605 and 0.68 . When the excitation force is $\in(0.74,1.00)$, the system carries out the steady-state periodic movement. Table 3 shows the form of movement of excitation force in different areas.

Figures 10 and 11 show the offset oscillogram, phase plane trace chart, and Poincaré diagram of the system when the excitation forces are 0.3 and 0.4 . At this time, it can be obtained that the offset oscillograms in Figures 10(a) and 11(a) have an irregular distribution with an unstable period. The phase trajectory charts in Figures 10(b) and 11(b) fill the phase space area and are neither overlapping nor entangled. The Poincaré diagrams in Figures 10(c) and 11(c) are neither a limited point set nor a closed curve, which indicates that the system is in the state of chaotic movement. 


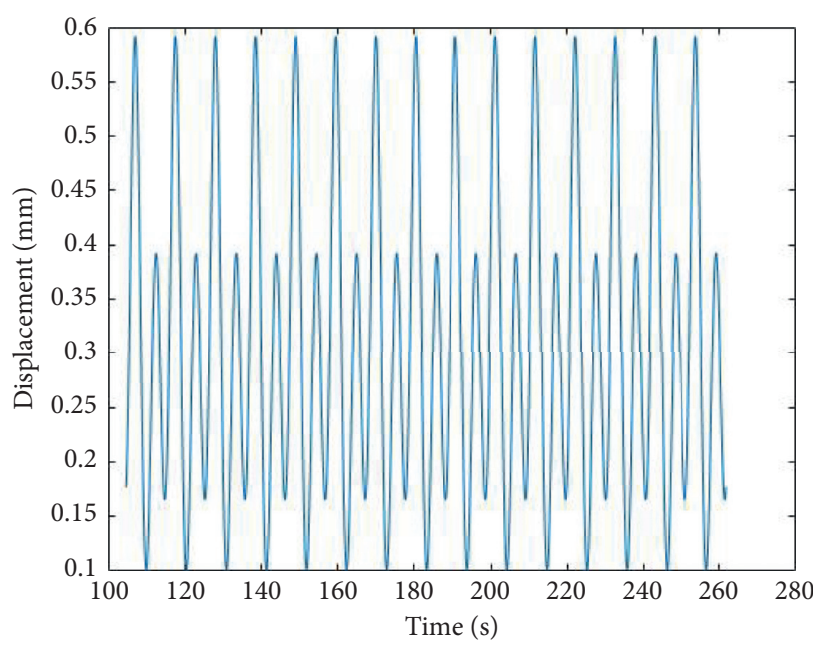

(a)

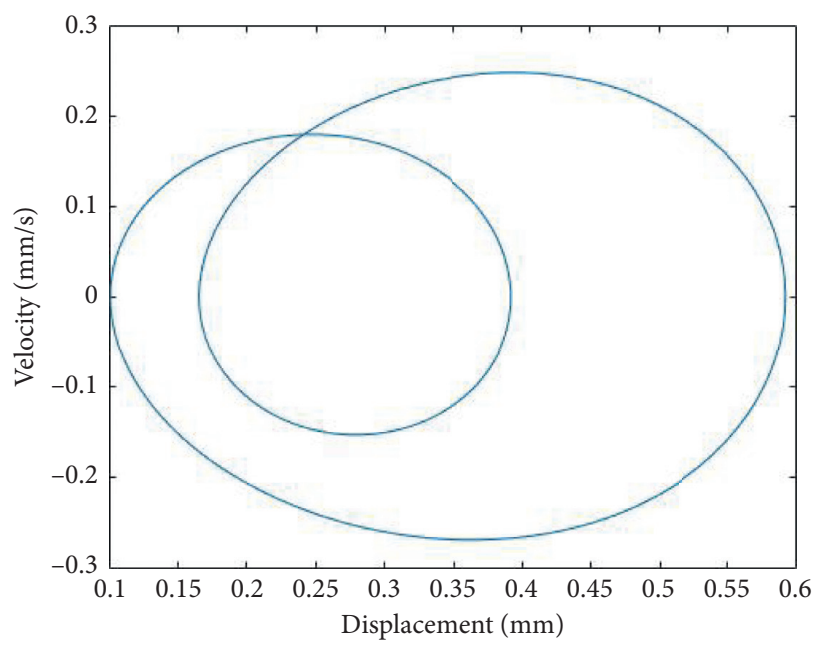

(b)

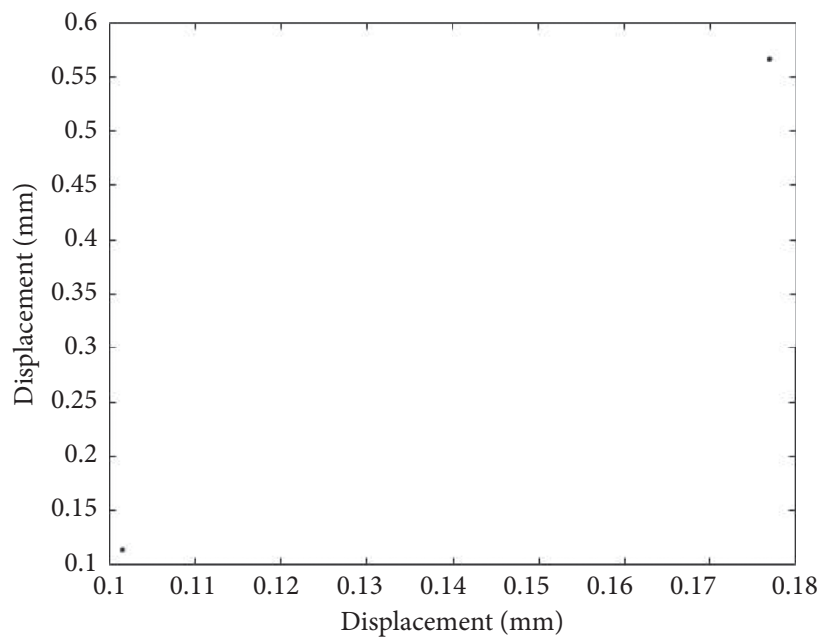

(c)

Figure 6: (a) The offset oscillogram, (b) phase trajectory chart, and (c) Poincaré diagram of the system when $C=0.2$.

5.3. The Influence of Exciting Frequency on the Chaotic Peculiarities of the System. Figure 12 shows the bifurcation chart of the GMA magnetic-mechanical coupled system changing with the exciting frequency. The initial condition is $\left[\begin{array}{lll}0 & 0 & 0.12\end{array}\right]$. When the exciting frequency changes, the path to chaos of the system is as follows:

Chaotic movement $\longrightarrow$ intermittent chaos $\longrightarrow$ periodic movement $\longrightarrow$ chaotic movement $\longrightarrow$ intermittent chaos $\longrightarrow$ periodic movement $\longrightarrow$ skip movement $\longrightarrow$ quasi - periodic movement $\longrightarrow$ chaotic movement $\longrightarrow$ multiple intermittent chaos $\longrightarrow$ periodic movement

We can see from Figure 12 that when the exciting frequency is $\epsilon(0.12,0.31)$, the system carries out the chaotic movement. When the exciting frequency is $\epsilon(0.31,0.36)$, the system carries out the movement with the period of 1 . When the exciting frequency is $\epsilon(0.36,0.42)$, the system carries out the chaotic movement, as shown in Figure $13(\omega=0.39)$, and has the intermittent chaos at the exciting frequency of 0.395 . When the exciting frequency is $\in(0.42,0.48)$, the system carries out the steady-state movement with the single period. When the exciting frequency is greater than 0.48 , the system carries out the skip movement. When the exciting frequency is $\epsilon(0.48,0.525)$, the system skips from the single period movement to quasiperiodic movement. When the exciting frequency is $\epsilon(0.525$, $0.655)$, the system carries out the chaotic movement, as shown 


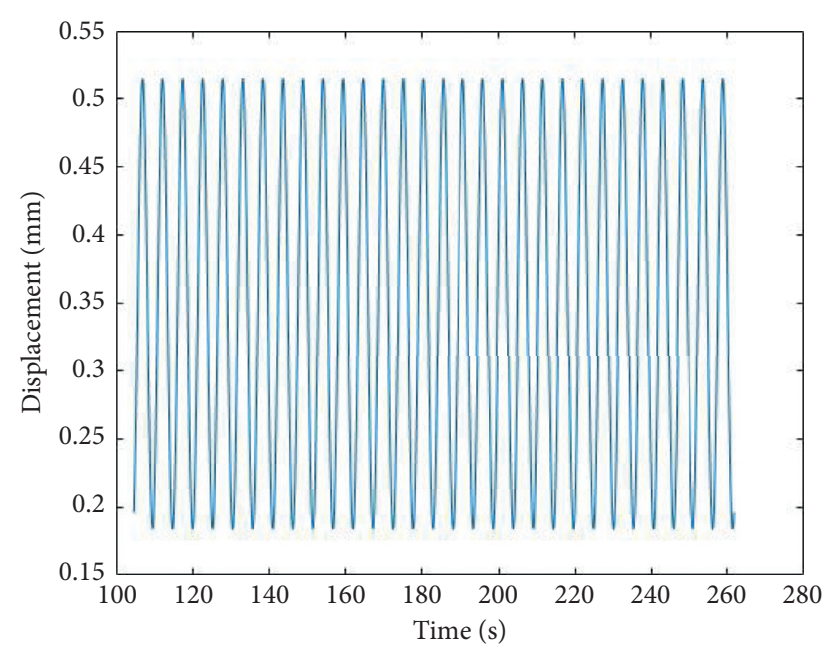

(a)

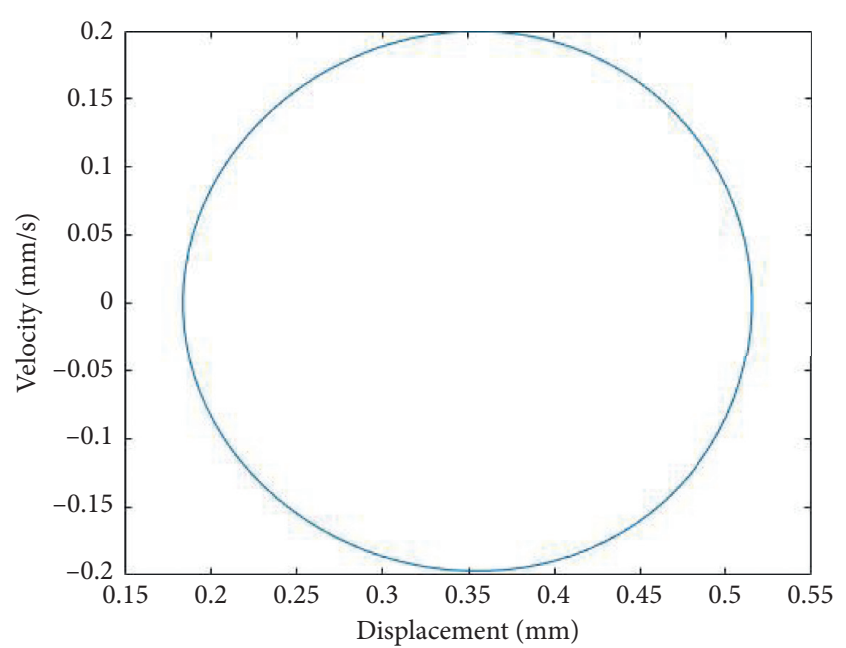

(b)

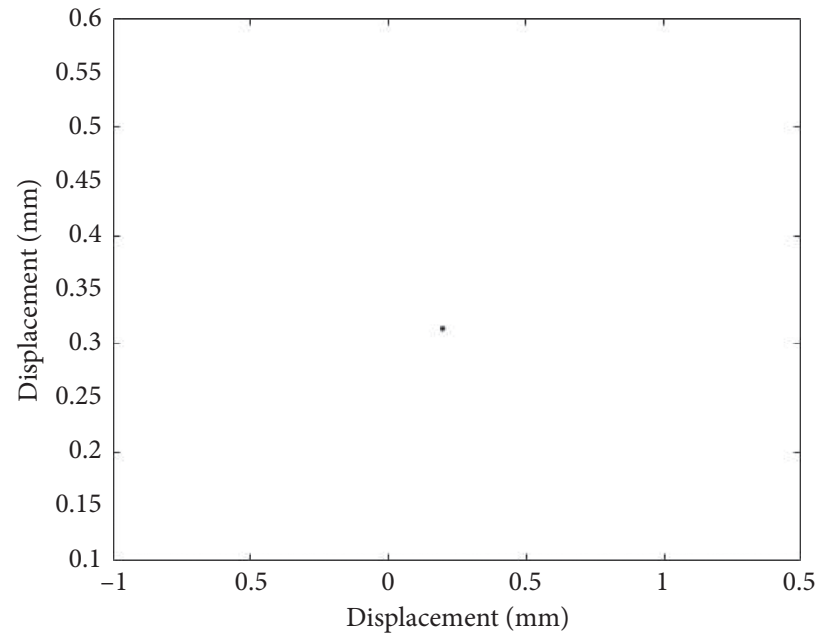

(c)

Figure 7: (a) The offset oscillogram, (b) phase trajectory chart, and (c) Poincaré diagram of the system when $C=0.5$.

TABLE 2: The form of movement of damping factor in different areas.

\begin{tabular}{lc}
\hline Ranges of $C$ & Movement form \\
\hline$(0.06,0.14)$ & Chaotic movement (Figure 5) \\
$(0.14,0.32)$ & Period 2 (Figure 6) \\
$(0.32,1.00)$ & Periodic movement (Figure 7) \\
\hline
\end{tabular}

in Figure $14(\omega=0.6)$. When the exciting frequency is $\in(0.655$, $1.00)$, the system carries out the steady-state movement with the single period, as shown in Figure $15(\omega=0.8)$, and has the intermittent chaos at the excitation frequencies around 0.665 , 0.69 , and 0.77 . Table 4 shows the form of movement of exciting frequency in different areas.

Figures 13 and 14 show the offset oscillogram, phase plane trace chart, and Poincaré diagram of the system when the excitation frequencies are 0.39 and 0.6 . It can be obtained that the offset oscillograms in Figures 13(a) and 14(a) have an irregular distribution with an unstable

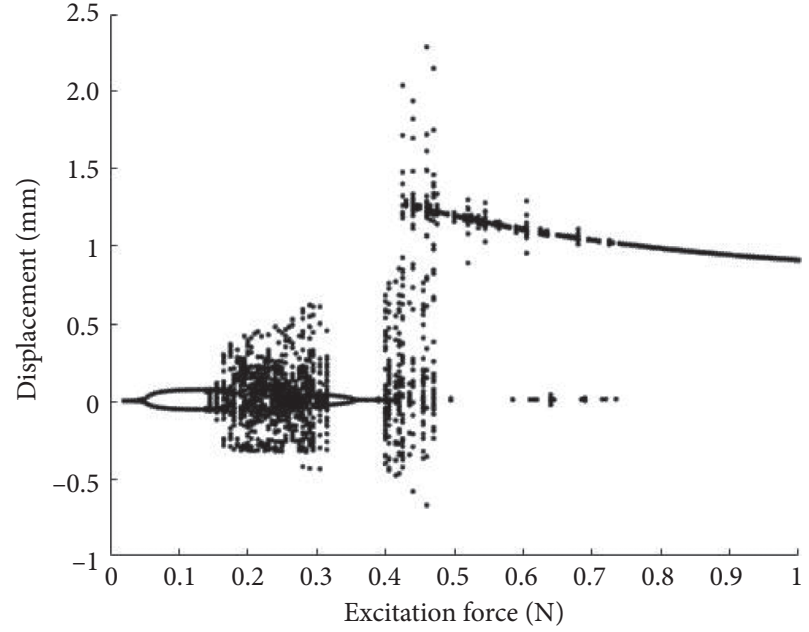

Figure 8: The bifurcation chart of the GMA magnetic-mechanical coupled system changing with the excitation force. 


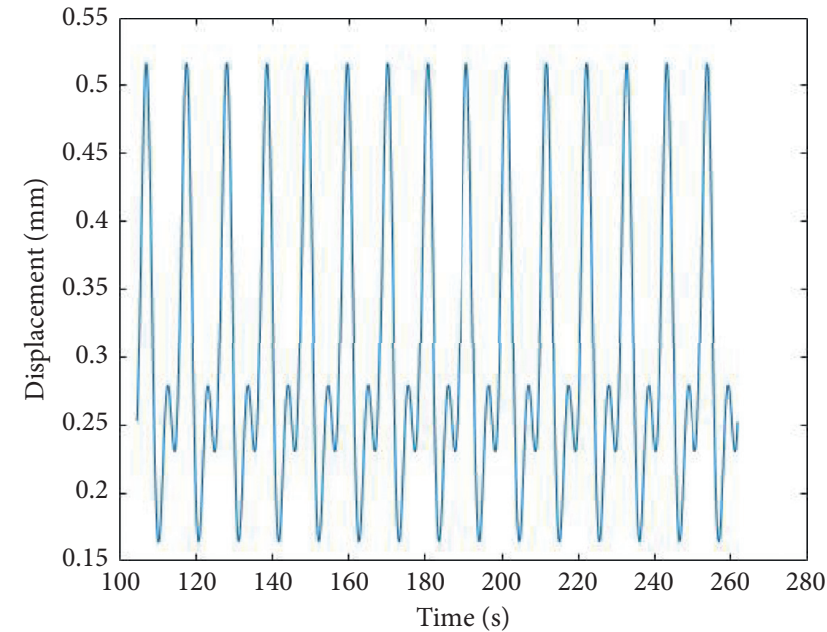

(a)

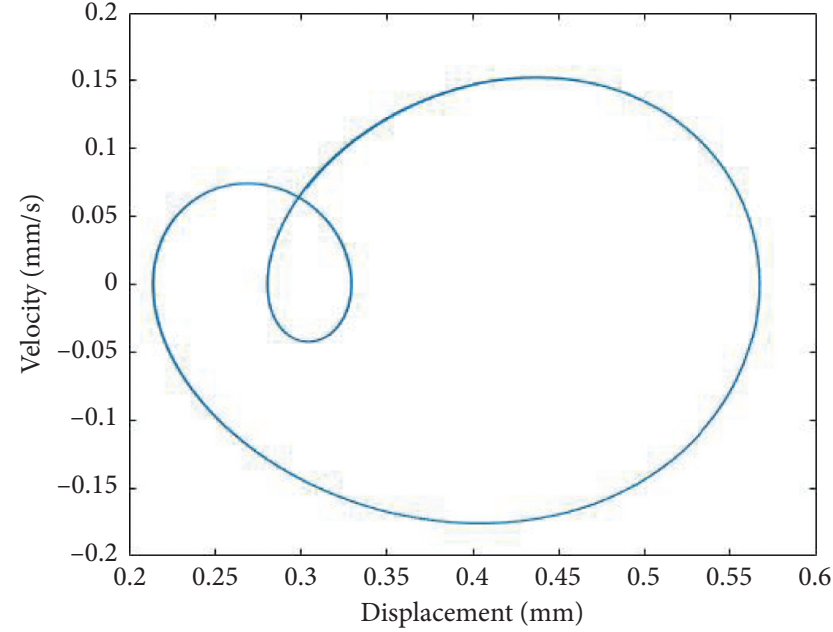

(b)

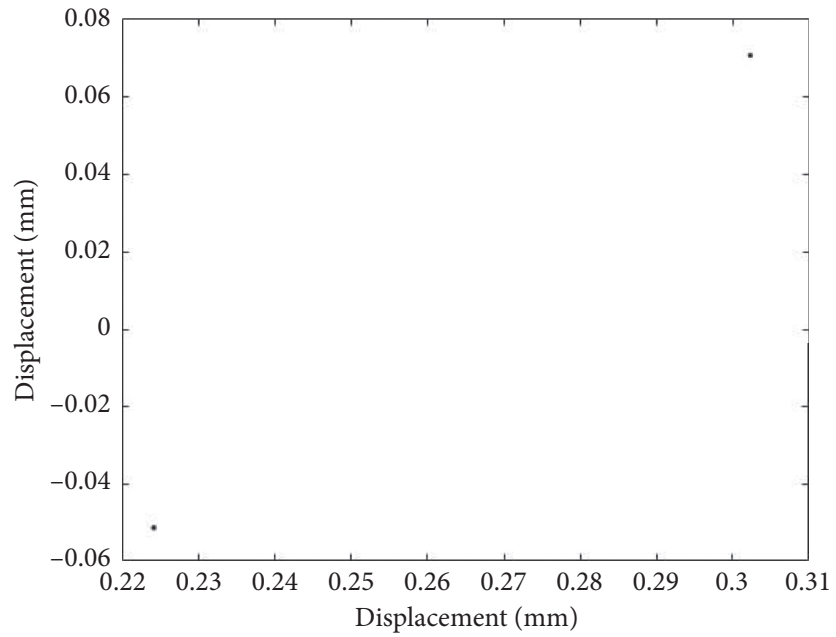

(c)

Figure 9: (a) The offset oscillogram, (b) phase trajectory chart, and (c) Poincaré diagram of the system when $F=0.1$.

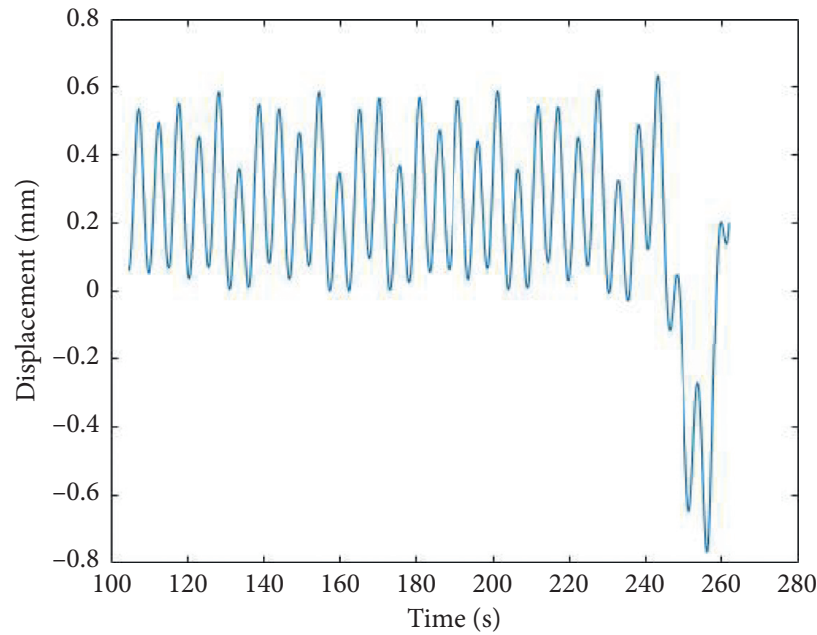

(a)

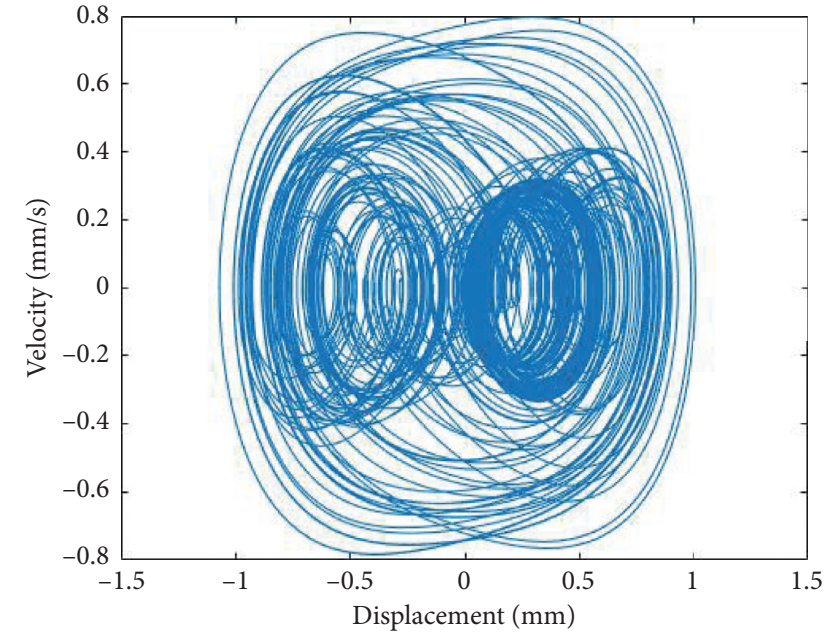

(b)

Figure 10: Continued. 


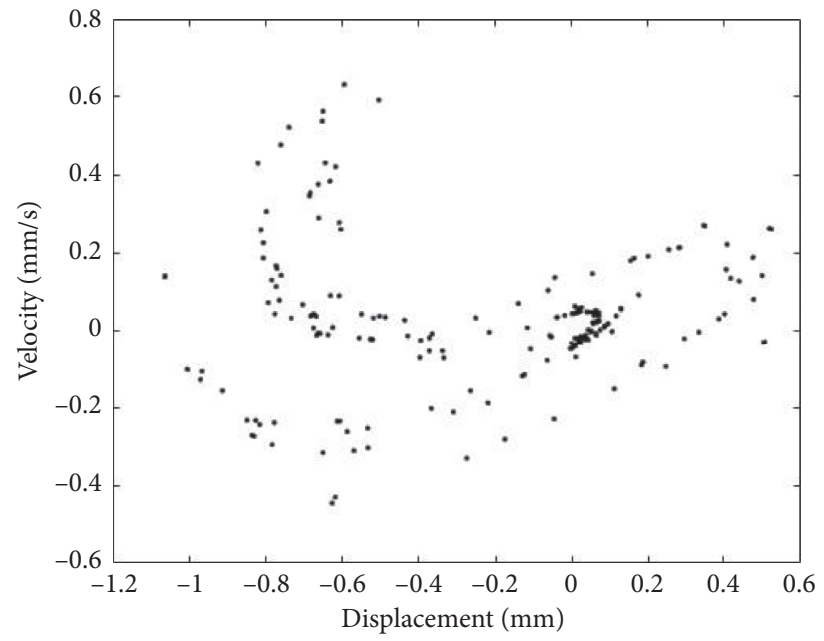

(c)

Figure 10: (a) The offset oscillogram, (b) phase trajectory chart, and (c) Poincaré diagram of the system when $F=0.3$.

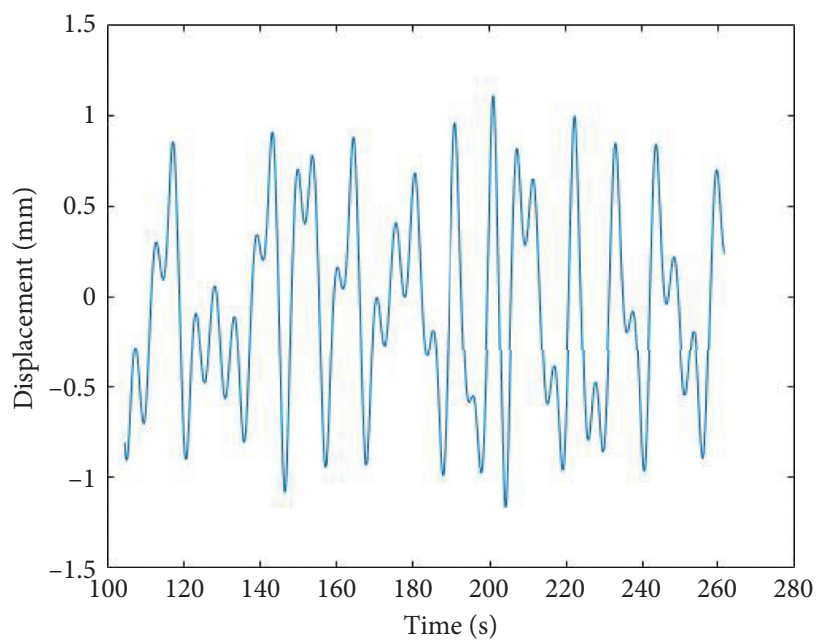

(a)

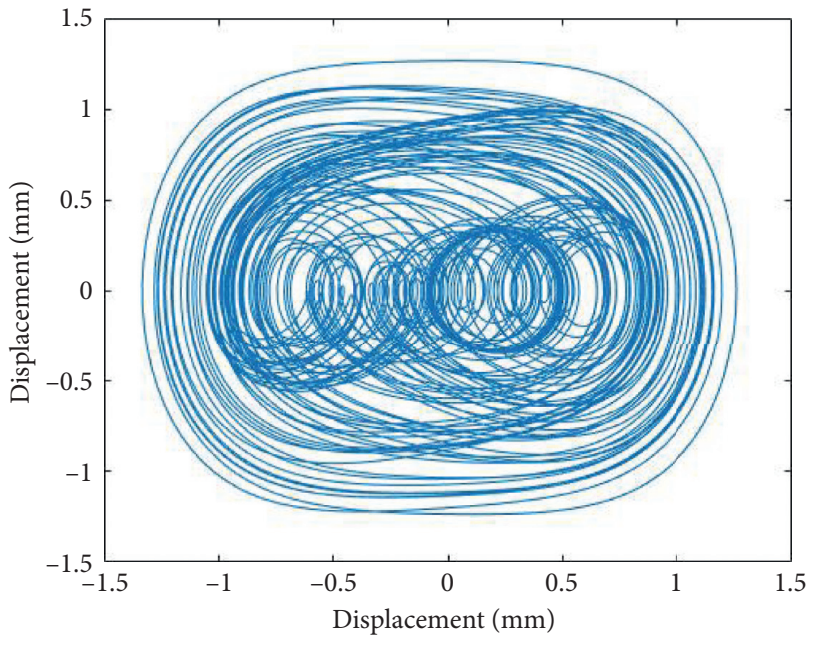

(b)

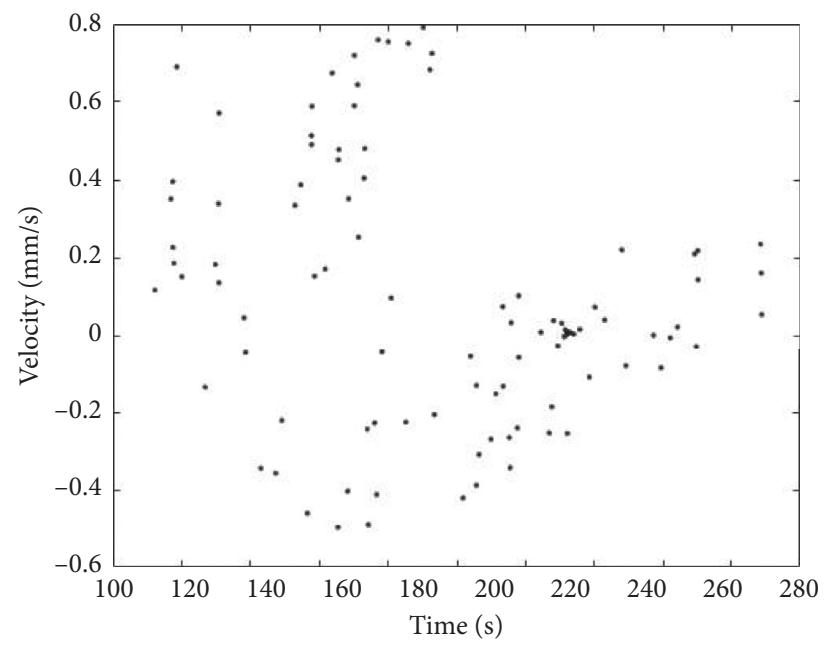

(c)

Figure 11: (a) The offset oscillogram, (b) phase trajectory chart, and (c) Poincaré diagram of the system when $F=0.4$. 
TABLE 3: The form of movement of excitation force in different areas.

\begin{tabular}{lc}
\hline Ranges of $F$ & Movement form \\
\hline$(0.02,0.045)$ & Periodic movement \\
$(0.045,0.14)$ & Period 2 (Figure 9) \\
$(0.14,0.315)$ & Chaotic movement (Figure 10) \\
$(0.315,0.36)$ & Period 2 \\
$(0.36,0.39)$ & Periodic movement \\
$(0.39,0.59)$ & Chaotic movement (Figure 11) \\
$(0.59,0.74)$ & Quasiperiodic movement \\
$(0.74,1.00)$ & Periodic movement \\
\hline
\end{tabular}

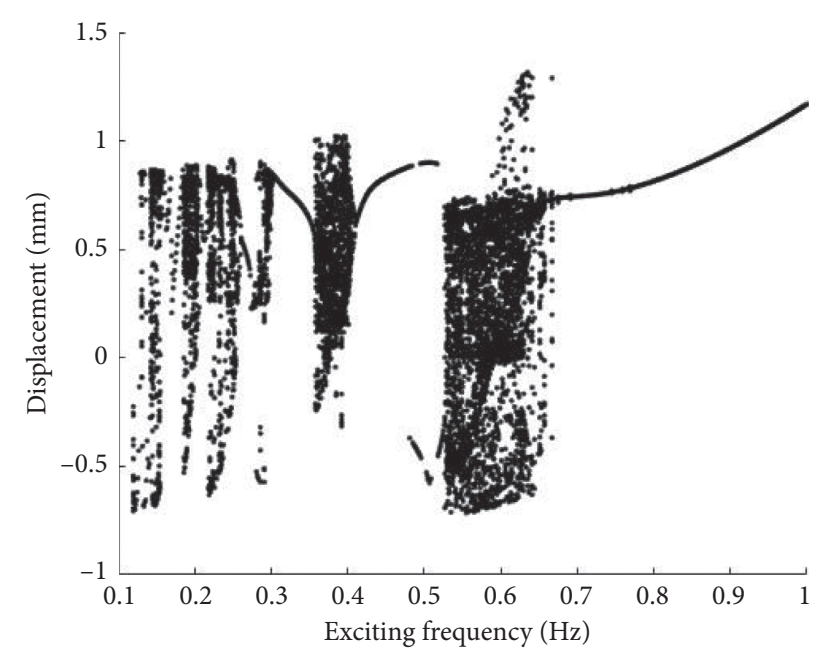

FIgURE 12: The bifurcation chart of the GMA magnetic-mechanical coupled system changing with the exciting frequency.

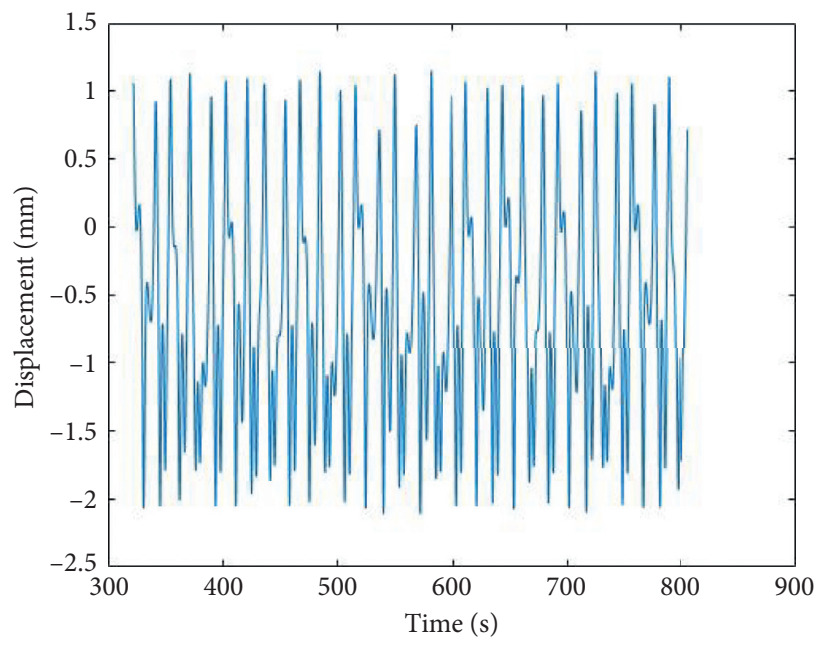

(a)

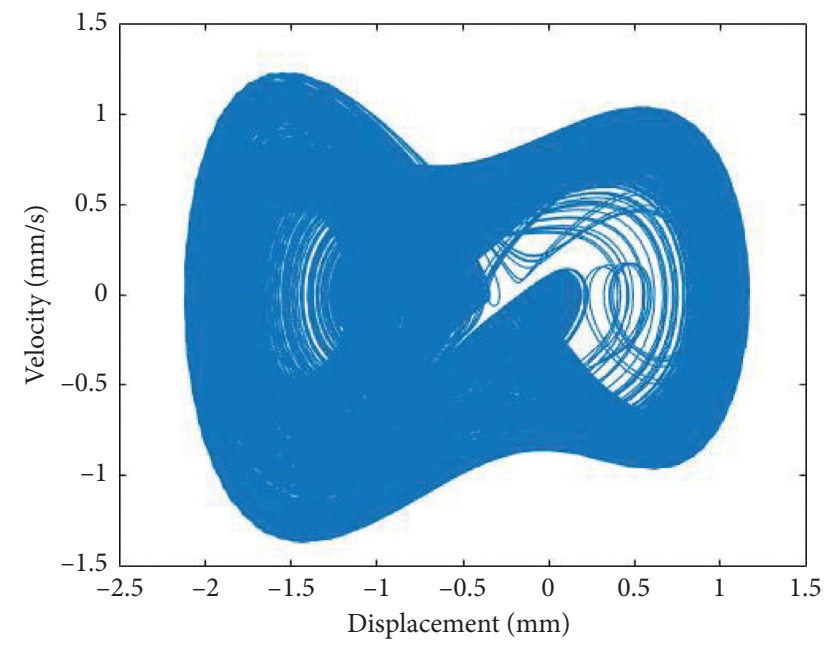

(b)

Figure 13: Continued. 


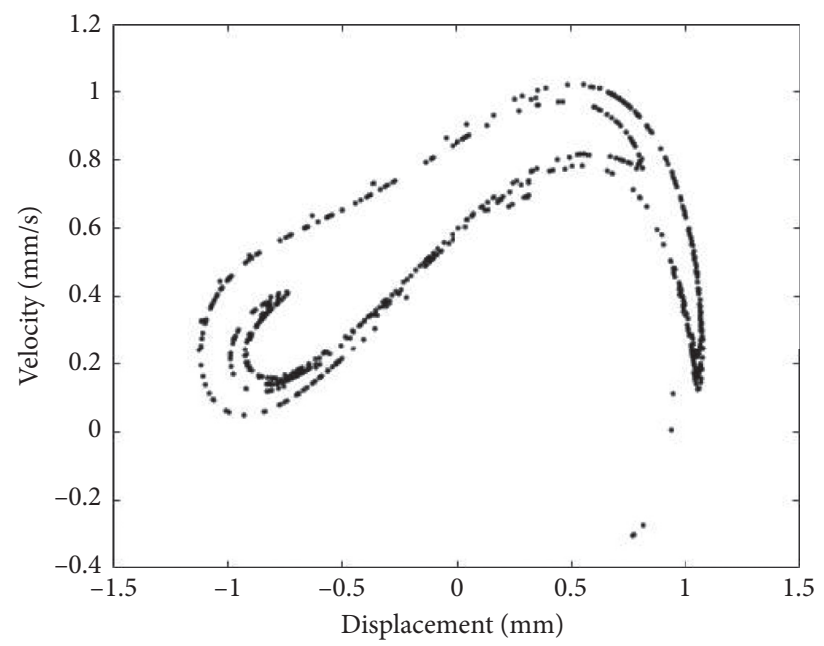

(c)

Figure 13: (a) The offset oscillogram, (b) phase trajectory chart, and (c) Poincaré diagram of the system when $\omega=0.39$.

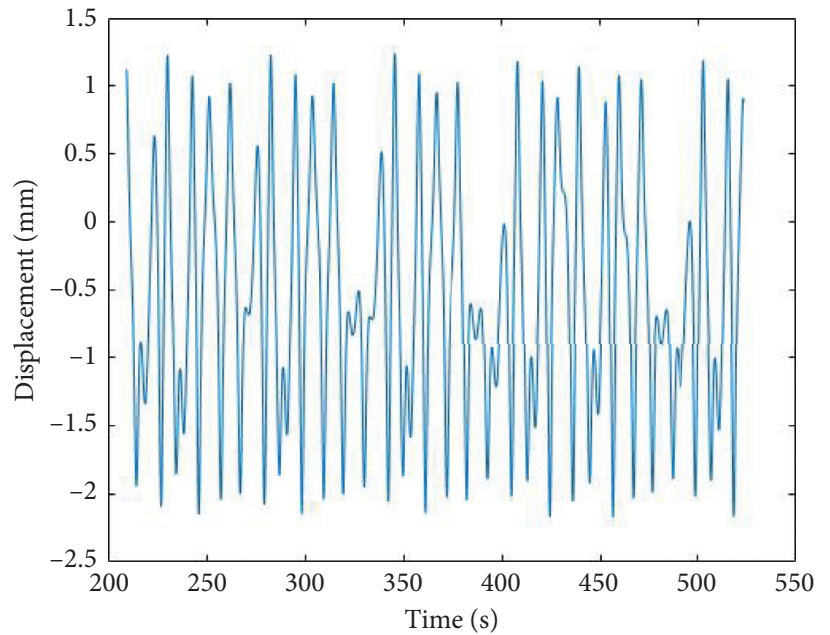

(a)

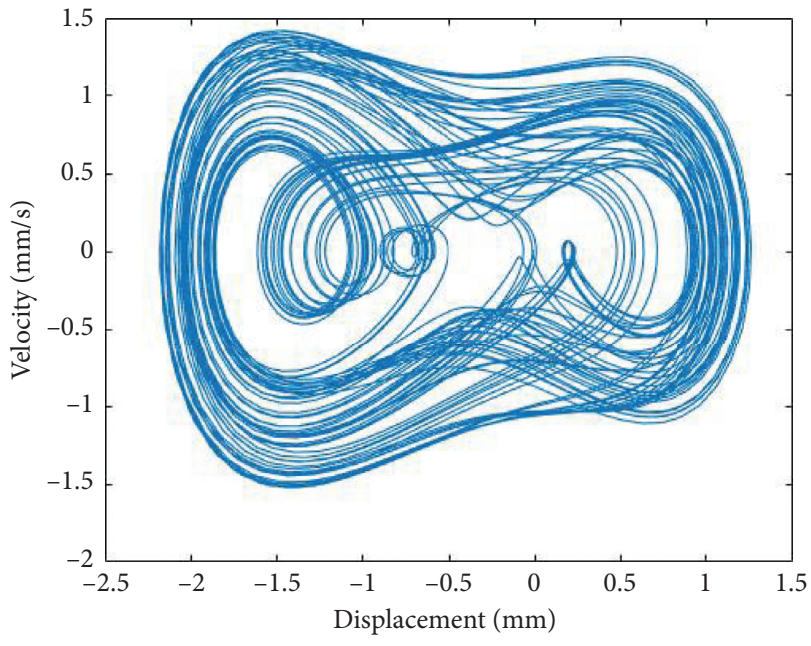

(b)

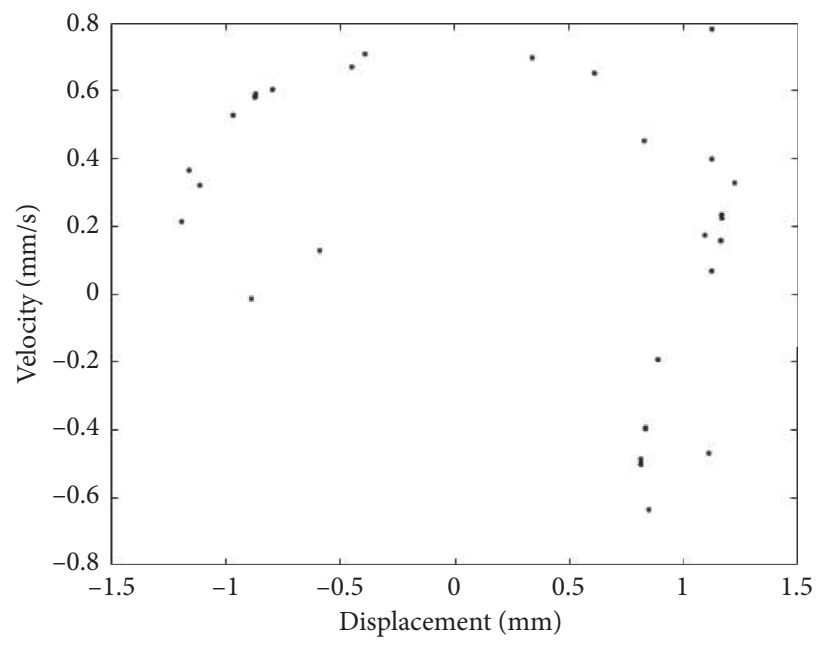

(c)

Figure 14: (a) The offset oscillogram, (b) phase trajectory chart, and (c) Poincaré diagram of the system when $\omega=0.6$. 


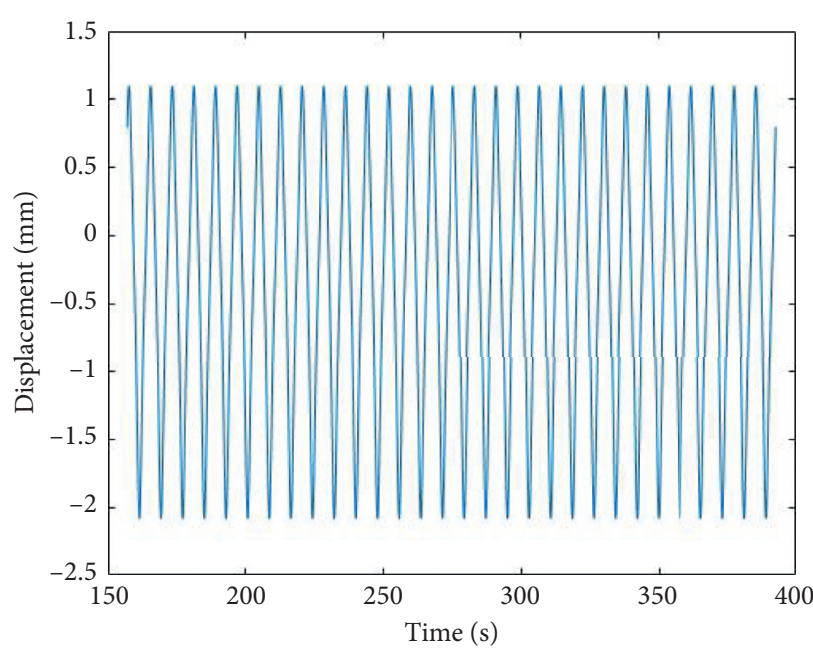

(a)

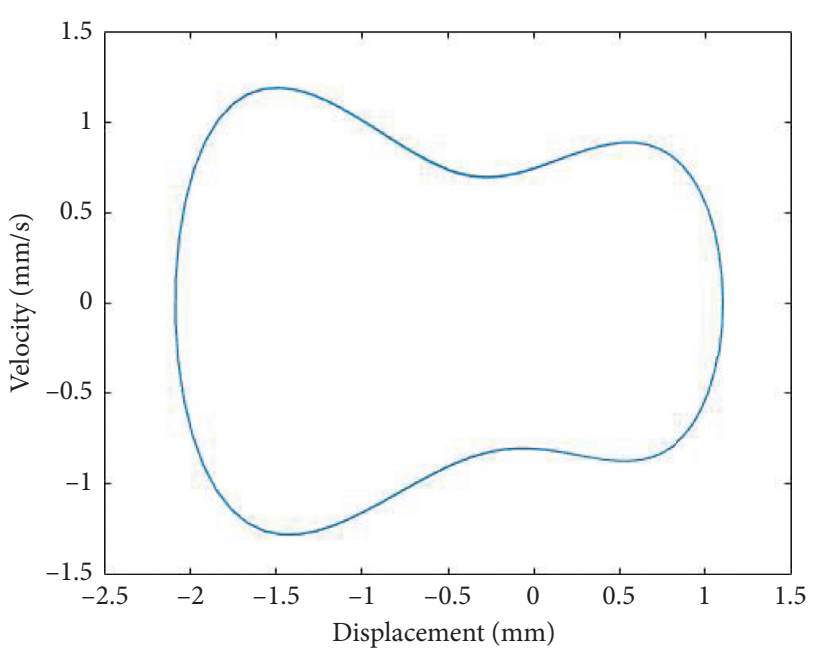

(b)

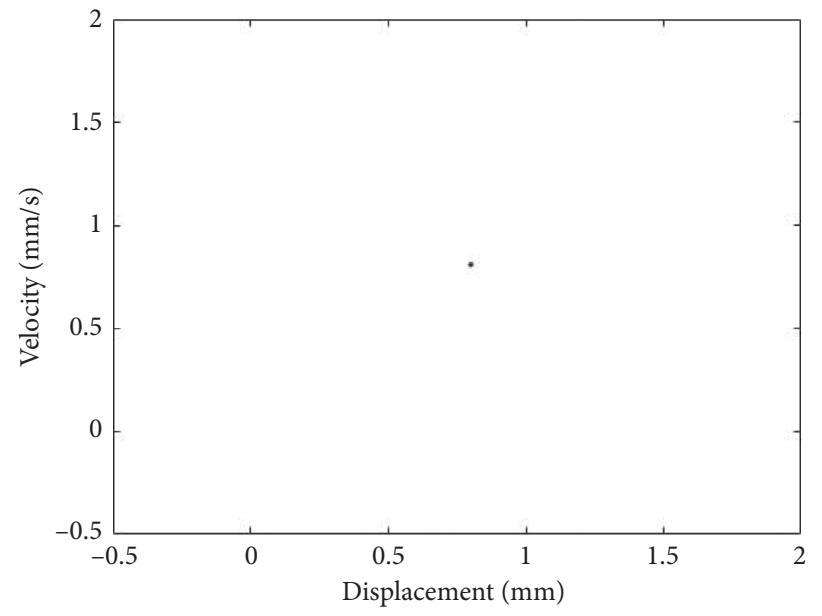

(c)

FIgURE 15: (a) The offset oscillogram, (b) phase trajectory chart, and (c) Poincare diagram of the system when $\omega=0.8$.

TABLE 4: The form of system movement of exciting frequency in different areas.

\begin{tabular}{lc}
\hline Ranges of $\omega$ & Movement form \\
\hline$(0.12,0.31)$ & Chaotic movement \\
$(0.31,0.36)$ & Periodic movement \\
$(0.36,0.42)$ & Chaotic movement (Figure 13) \\
$(0.42,0.48)$ & Periodic movement \\
$(0.48,0.525)$ & Quasiperiodic movement \\
$(0.525,0.655)$ & Chaotic movement (Figure 14) \\
$(0.655,1.00)$ & Periodic movement (Figure 15) \\
\hline
\end{tabular}

period. The phase trajectory charts in Figures 13(b) and 14(b) fill the phase space region and are neither overlapping nor entangled. The Poincaré diagrams in Figures 13(c) and 14(c) are neither a finite point set nor a closed curve, which proves that the system is in the state of chaotic movement.

We can see from Figure 15 that when the exciting frequency is 0.8 , the offset oscillogram has a regular distribution with a constant period. The phase trajectory chart is a closed curve, and Poincaré diagram shows an attractive point, which indicates that the system carries out a typical steady-state movement with the single period.

\section{Coupled System with Parametric Errors}

In order to study the influence of parametric uncertainties on the coupled system, the parameters can be compared with the random errors within the range of $\pm 20 \%$, which is similar to the method adopted in [31-33]. Take Figure 15(b) as an example; when $\omega$ is the random error within the range of $\pm 20 \%$, as shown in Figure 16, the amplitude of displacement decreases by $0.18 \%$, and a similar periodic orbit also exists. The results show that the coupled system can stay on a periodic orbit with more sensitive parameters. It indicates that the time interval of $\omega$ leads the system to periodicity motion. This is also similar to the conclusion from Tusset et al. [31]. Jia et al. [34] designed one self-adaptive algorithm based on the Preisach model and processed dynamic adjustment at the parameters; this algorithm validly 


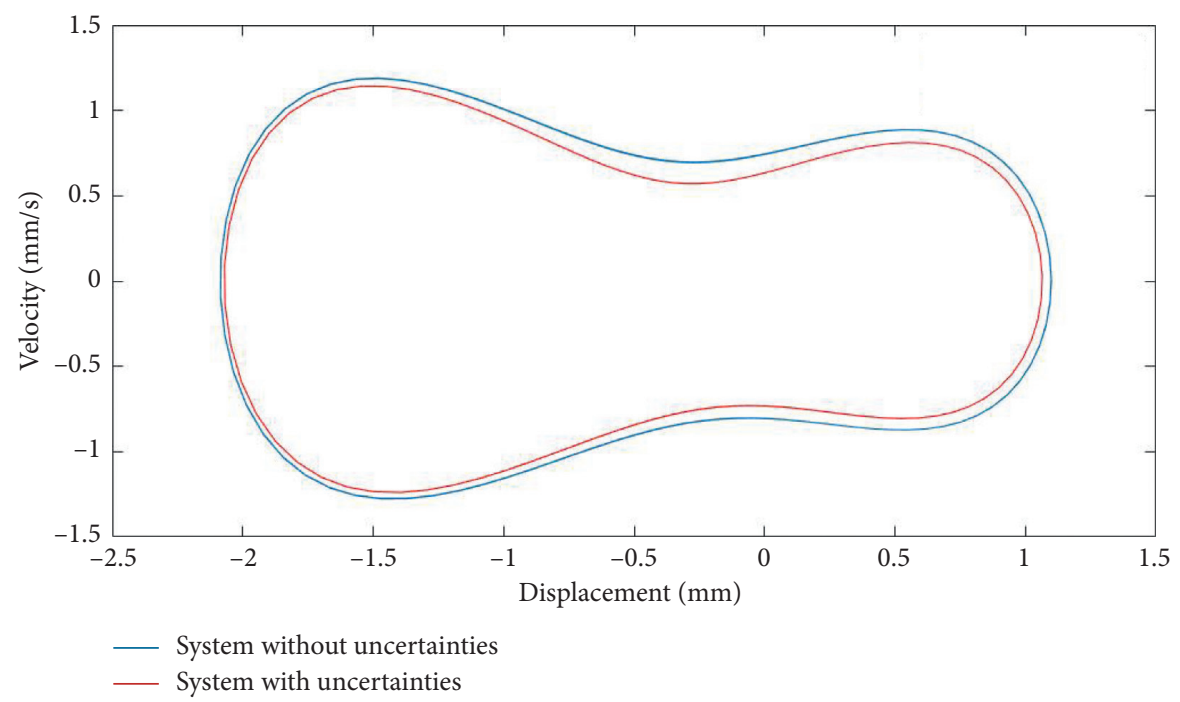

FIGURE 16: Coupled system without and with parametric uncertainties.

compensated nonlinear hysteresis of output displacement of the giant magnetostrictive actuator, reduced the parameter errors of the system, and also improved the control precision of the actuator.

To make giant magnetostrictive magnetic-mechanical coupled system enter into periodicity motion, we can lead a passive rotational magnetorheological (MR) damper into the system. The MR damper restrains the chaos action of the system through adding different currents. The research in [35] found that the simple introduction of a passive rotational MR damper without electric current has a smaller effect on the chaos action of the system, so we could use the rotational MR damper with energizing discontinuity to make the coupled system keep periodicity vibration. Tusset et al. [31] processed the state-dependent Riccati equation (SDRE) control and the nonlinear saturation control (NSC) which are related to the status of the system; it could also make the coupled system enter into periodicity status.

\section{Conclusion}

(1) Chaotic peculiarities of the GMA magnetic-mechanical coupled system were studied, and the value range of each parameter in chaotic movement of the system was obtained through numerical modeling: chaotic movement occurs when the damping factor of the system is within the interval of $(0.06,0.14)$ or when the excitation force is within the intervals of $(0.14,0.315)$ and $(0.39,0.59)$ or when the exciting frequency is within the intervals of $(0.12,0.31),(0.36$, $0.42)$ and $(0.525,0.655)$.

(2) By controlling the damping factor, excitation force, and exciting frequency parameters of the system, the GMA magnetic-mechanical coupled system can avoid chaotic movement purposefully, thus improving the stability of GMA.

(3) The chaotic peculiarities of the GMA magneticmechanical coupled system were subjected to theoretical analysis, which could effectively avoid the occurrence of chaotic movement, improve the stability of the GMA system, and contribute to the wide application of GMA.

\section{Data Availability}

The data used to support the findings of this study are available from the corresponding author upon request.

\section{Conflicts of Interest}

The authors declare that there are no conflicts of interest regarding the publication of this paper.

\section{References}

[1] J. Liu, C. Jiang, and H. Xu, "Giant magnetostrictive materials," Science China Technological Sciences, vol. 55, no. 5, pp. 1319-1326, 2012.

[2] F. Claeyssen, N. Lhermet, R. L. Letty et al., "Actuators, transducers and motors based on giant magnetostrictive materials," Journal of Alloys and Compounds, vol. 258, no. 1-2, pp. 0-73, 1997.

[3] H. B. Yan, H. Gao, H. B. Hao et al., "Design and simulation of excitation coil of rare earth giant magnetostrictive actuator," Mechanical Science and Technology for Aerospace Engineering, vol. 38, no. 10, pp. 1569-1575, 2019.

[4] X. H. Zhang, Study on Crucial Technology of Active Vibration Isolation System Based on Structure-Optimized Giant Magnetostrictive Actuator, Beihang University, Beijing, China, 2008.

[5] H. Zhang, T. Zhang, and C. Jiang, "Magnetostrictive actuators with large displacement and fast response," Smart Materials and Structures, vol. 21, no. 5, pp. 55014-55020, 2012.

[6] Z.-Y. Jia, H.-F. Liu, F.-J. Wang, and C.-Y. Ge, "Research on a novel force sensor based on giant magnetostrictive material and its model," Journal of Alloys and Compounds, vol. 509, no. 5, pp. 1760-1767, 2011.

[7] S.-J. Moon, C.-W. Lim, B.-H. Kim, and Y. Park, "Structural vibration control using linear magnetostrictive actuators," 
Journal of Sound and Vibration, vol. 302, no. 4-5, pp. 875-891, 2007.

[8] G. Xue, P. Zhang, X. Li et al., "A review of giant magnetostrictive injector (GMI)," Sensors \& Actuators A Physical, vol. 273, pp. 159-181, 2018.

[9] B. Yoo, M. Hirano, and K. Hirata, "Fully coupled electromagneto-mechanical analysis method of magnetostrictive actuator using 3-D finite element method," in Proceedings of the 2008 18th International Conference on Electrical Machines, September 2008.

[10] M. Niu, B. Yang, Y. Yang, and G. Meng, "Dynamic modelling of magnetostrictive actuator with fully coupled magnetomechanical effects and various eddy-current losses," Sensors and Actuators A: Physical, vol. 258, pp. 163-173, 2017.

[11] H. Ebrahimi, Y. Gao, A. Kameari, H. Dozono, and K. Muramatsu, "Coupled magneto-mechanical analysis considering permeability variation by stress due to both magnetostriction and electromagnetism," IEEE Transactions on Magnetics, vol. 49, no. 5, pp. 1621-1624, 2013.

[12] Q. Yang, R. Yan, C. Fan, H. Chen, F. Liu, and S. Liu, "A magneto-mechanical strongly coupled model for giant magnetostrictive force sensor," IEEE Transactions on Magnetics, vol. 43, no. 4, pp. 1437-1440, 2007.

[13] P. Zhao, Experimental Investigations of Magneto-Mechanical Coupled Characteristics in Giant Magnetos-Trictive Materials, Lanzhou University, Lanzhou, China, 2012.

[14] G. P. Li, Study on Active Vibration Isolative Techniques for Precise Instruments, Zhejiang University, Hangzhou, China, 2010.

[15] S. Y. Cao, Dynamic Model with Hystersis Nonlinearity and Control Technique for Giant Magnetostrictive Actuator, Hebei University of Technology, Tianjin, China, 2004.

[16] Z. Zhu, Q. Zhang, and J. Xu, "Stochastic bifurcation and fractal and chaos control of a giant magnetostrictive filmshape memory alloy composite cantilever plate subjected to in-plane harmonic and stochastic excitation," Journal of Applied Physics, vol. 115, 2014.

[17] J. Xu, R. Li, and Z. Zhu, "Nonlinear response characteristics of giant magnetostrictive-piezoelectric composite sensors," Sensors \& Materials, vol. 27, no. 8, pp. 633-639, 2015.

[18] S. Bowong and F. M. M. Kakmeni, "Chaos control of uncertain chaotic systems via backstepping approach," Journal of Vibration \& Acoustics, vol. 128, no. 1, pp. 1007-1024, 2006.

[19] R. Sarmah and G. Ananthakrishna, "Dynamics of strain bifurcations in a magnetostrictive ribbon," Physical Review E, vol. 86, no. 1, 2012.

[20] H. G. Sun, Coupled Magneto-Elastic Theory of Giant Magnetostrictive Transducer and Application in Cutti- $\mathrm{Ng} \mathrm{Ma-}$ chining, Northeastern University, Shenyang, China, 2008.

[21] H.-B. Yan, H. Gao, G.-W. Yang, H.-B. Hao, Y. Niu, and P. Liu, "Bifurcation and chaos characteristics of hysteresis vibration system of giant magnetostrictive actuator," Chinese Physics B, vol. 29, no. 2, p. 020504, 2020.

[22] M. Yao, W. Zhang, and J. W. Zu, "Multi-pulse chaotic dynamics in non-planar motion of parametrically excited viscoelastic moving belt," Journal of Sound and Vibration, vol. 331, no. 11, pp. 2624-2653, 2012.

[23] Y. Li, H. Bai, Z. He et al., "Chaotic characteristics of nonlinear metal rubber vibration isolation system," Zhongguo Jixie Gongcheng/China Mechanical Engineering, vol. 26, no. 14, pp. 1871-1876, 2015.

[24] Z. Wei, W. Zhang, and M. Yao, "On the periodic orbit bifurcating from one single non-hyperbolic equilibrium in a chaotic jerk system," Nonlinear Dynamics, vol. 82, no. 3, pp. 1251-1258, 2015.

[25] R. Q. Wu, W. Zhang, and M. H. Yao, "Nonlinear dynamics near resonances of a rotor-active magnetic bearings system with 16-pole legs and time varying stiffness," Mechanical Systems and Signal Processing, vol. 100, pp. 113-134, 2018.

[26] Y. Chai, Z. Song, and F. M. Li, "Nonlinear vibrations, bifurcations and chaos of lattice sandwich composite panels on winkler-pasternak elastic foundations with thermal effects in supersonic airflow," Meccanica, vol. 54, pp. 919-944, 2019.

[27] D. M. Dozor, M. J. Gerver, and J. R. Swenbeck, "Nonlinear modeling for control of Terfenol-D based actuators," in Proceedings of the SPIE International Society for Optical Engineering, San Diego, CA, USA, March 1997.

[28] Q. Lin, G. X. Zhang, Q. W. He et al., "Modeling of giant magnetostrictive actuators," Mechatronics, vol. 17, no. 6, pp. 26-29, 2001.

[29] T. Z. Liu, B. M. Li, B. Ye et al., "Complex profile of engine piston skirt produced by a technology with vibration," Journal of Xi'an Jiaotong University, no. 10, pp. 3-5, 1998.

[30] W. S. Lu, Computational Design and Manufacturing of Disc Spring, Fudan University Press, Shanghai, China, 1990.

[31] A. M. Tusset, V. Piccirillo, A. M. Bueno et al., "Chaos control and sensitivity analysis of a double pendulum arm excited by an RLC circuit based nonlinear shaker," Journal of Vibration \& Control, vol. 22, no. 7, 2015.

[32] A. M. Tusset, V. Piccirillo, J. M. Balthazar, and R. M. L. R. da Fonseca, "On suppression of chaotic motions of a portal frame structure under non-ideal loading using a magneto-rheological damper," Journal of Theoretical and Applied Mechanics, vol. 53, no. 3, p. 653, 2015.

[33] R. H. Avano, A. M. Tusset, J. M. Balthazar et al., "On nonlinear dynamics behavior of an electro-mechanical pendulum excited by a nonideal motor and a chaos control taking into account parametric errors," Journal of the Brazilian Society of Mechanical Ences and Engineering, vol. 40, no. 1, p. 23, 2018.

[34] Z. Jia, F. J. Wang, J. Zhang et al., "Hysteresis nonlinearity mode-ling and control of giant magnetostrictive actuator," Chinese Journal of Mechanical Engineering, vol. 41, no. 7, pp. 131-135, 2005.

[35] A. M. Tusset, F. C. Janzen, V. Piccirillo et al., "On nonlinear dynamics of a parametrically excited pendulum using both active control and passive rotational (MR) damper," Journal of Vibration and Control, vol. 24, no. 9, 2017. 